\title{
$\operatorname{arCOS} D E S I G N$
}

\section{A representação gráfica da gestão de design aliada ao desenvolvimento artesanal de uma comunidade criativa}

Erik Silva dos Santos (UFSC, Brasil)

dossantos.erik@gmail.com

Campus Universitário Reitor João David Ferreira Lima, sala 122 Bloco A Trindade, Florianópolis, SC, Brasil CEP: 88040-970

Luiz Fernando Gonçalves de Figueiredo (UFSC, Brasil)

lffigueiredo2009@gmail.com

Giselle Schmidt Alves Diaz Merino (UFSC, Brasil)

gisellemerino@gmail.com 


\title{
A representação gráfica da gestão de design aliada ao desenvolvimento artesanal de uma comunidade criativa
}

Resumo: O design no processo de desenvolvimento de produtos é elemento essencial na definição planejada daquilo que comunica uma mensagem e do que vende. A gestão de design apresenta recursos e diretrizes necessárias para aumento da competitividade de um produto, muitas vezes promovendo inovação em seu eixo de mercado. O artesanato - que além de materializar a alma da cultura brasileira, é um setor da economia cujo crescimento possui alto potencial de geração de trabalho e renda, promove a preservação das culturas locais e a formação de uma mentalidade empreendedora. Partindo dessas premissas, ressalta-se a importância de se aliar a gestão de design ao artesanato, uma vez que promove a cultura local de um povo e torna o mesmo, autossuficiente financeiramente enquanto estrutura empreendedora. O presente artigo busca desenvolver a estrutura e apresentar a representação gráfica de como o design pode contribuir para a melhoria de uma identidade local direcionada ao artesanato, ou seja, buscar as relações e intersecções entre o design e o artesanato - a intenção é facilitar a aplicação dos conceitos de design aos artesãos e demais envolvidos em uma comunidade ou grupo criativo de forma simplificada e ilustrada, que possa ser compreendida universalmente e que, acima de tudo auxilie essas comunidades ou grupos criativos a representarem através de seus produtos, a identidade que lhes representa.

Palavras-chave: Gestão de Design, Artesanato, Identidade, Cultura, Representação Gráfica.

\section{The graphical representation of design management allied to the craft development of a community creative}

\begin{abstract}
The design on process in product development is an essential element in the definition of what planned and communicates a message that sells. The management of design provides resources and guidance necessary to increase the competitiveness of a product, often promoting innovation on its axis market. The craft - which besides materialize the soul of Brazilian culture, is an industry whose growth has high potential to generate employment and income, promotes the preservation of local cultures and the formation of an entrepreneurial mindset. Based on these assumptions, it emphasizes the importance of combining the management of design to craft, since it promotes the local culture of a people and makes it, financially self-sufficient while entrepreneurial structure. This paper presents a framework and present
\end{abstract}


a graphical representation of how design can contribute to the improvement of a local identity directed the craft, ie, looking for relationships and intersections between design and craftsmanship - the intention is to facilitate the application of design concepts to artisans and others involved in a community or group creative and illustrated in simplified form, that can be universally understood and above all assist these communities and creative groups to represent through their products, the identity that represents them.

Key words: Management of Design, Craft, Identity, Culture, Graphical representation. 


\section{Introdução}

A importância do design em qualquer âmbito é notável, desde as recomendações ergonômicas em um posto de trabalho, até a identidade visual do produto desenvolvido no referido posto. Apesar disso, ainda há limitação na implementação do design como ferramenta de melhoria em setores como o artesanato - que além de materializar a alma da cultura brasileira, é um setor da economia cujo crescimento possui alto potencial de geração de trabalho e renda, merecendo uma política de desenvolvimento sustentável, levando à preservação das culturas locais e à formação de uma mentalidade empreendedora. Seguindo esta linha, o artigo busca pontuar e discutir a atuação da gestão de design no artesanato, uma vez que se busca a intervenção da gestão de design nesta área como alternativa para evidenciar a qualidade e a imagem do produto, além do uso da sustentabilidade como suporte para a extração e processo adequados de recursos na concepção do mesmo. O design no processo de desenvolvimento de produtos é elemento essencial na definição planejada daquilo que comunica uma mensagem e do que vende. A gestão de design apresenta recursos e diretrizes necessárias para aumento da competitividade de um produto em seu eixo de mercado, através da melhoria da produção, qualidade e melhoria da imagem. $\mathrm{O}$ design gráfico está ligado diretamente à imagem de um produto, a partir do primeiro contato do consumidor. Umas séries de perguntas apontam essa ligação: Que produto é este? (identidade visual); Como ele vem acondicionado? (embalagem); Como usar? (design de advertência, pictogramas e etc) entre outras.

É a partir dessas indagações, que este, apresenta a representação gráfica como alternativa para a melhoria da compreensão dos aspectos tangíveis e intangíveis que delimitarão essa relação da gestão do design com o artesanato.

\section{Por que relacionar a gestão de design ao artesanato?}

A cultura é fator condicionante nas relações sociais, intervém no meio social de forma dinâmica e pode modificar ou representar as características estruturais da sociedade. A cultura cumpre a função dentro das necessidades de produção, a função de reelaborar as estruturas sociais e sugerir outras novas - além de contribuir para essas relações de produção, contribui para a reprodução, transformação e criação de outras relações (CANCLINI, 1983).

As peças que compõem o artesanato são ricas em detalhes e são derivadas do conhecimento tácito do artesão. O artesanato regional está diretamente ligado a elementos da cultura local. 
A Gestão do Design tem como sua principal característica, a multidisciplinaridade e busca através disso, integrar o processo de desenvolvimento de um produto ou serviço, desde a criação ou concepção inicial até a comercialização do mesmo, visando atender as necessidades do consumidor e, além disso, satisfazer a organização e os seus colaboradores. Implementar a Gestão do Design consiste em uma mudança comportamental, através de trabalho co-participativo, estruturação de imagem corporativa e acima de tudo, significa estabelecer metas e diretrizes para que a comunidade tenha um plano de conduta a longo prazo.

A importância deste estudo se dá, uma vez que apesar do crescente número de pesquisas direcionadas a Gestão do Design, pouco ainda se fala sobre a inter-relação entre a Gestão do Design e o artesanato.

Além disso, o Brasil é um país com uma diversidade cultural gigantesca e são inúmeras as possibilidades de estudos direcionados a identidade cultural de uma região. A gestão de design busca se integrar a diversas realidades a fim de proporcionar melhores experiências e maximizar os resultados, assim a pesquisa se torna relevante uma vez que, o país de modo geral, tem um potencial significativo de produção artesanal e este polo tem uma demanda constante já que o turismo alavanca o setor; é de fundamental importância que a Gestão de Design não seja instrumento de intervenções pontuais no processo, para isso é necessário entender e mensurar em que níveis se podem atuar (seja estratégico, tático ou operacional) de forma que a contribuição para o artesanato.

Tomando o contexto histórico, sabemos quedo período paleolítico ao neolítico, cerca de 3.500 a.C. a 4.000 a.C., os povos faziam pinturas nas cavernas com princípios práticos e de ritual, em alguns casos, as marcas aparentavam serem feitas por artefatos como lanças. Nesse período a utilização da representação gráfica, sugeria o efeito de magia - uma vez que se buscava sucesso na caça e inspirava esforço para a cooperação no grupo. Atualmente, busca-se praticidade e facilidade na transmissão e na compreensão dos dados; fazer uso de ilustrações, esquemas, diagramas e infográficos são as melhores alternativas para que a mensagem seja passada de uma forma universal (sem distorções através das interpretações diversas) e eficaz.

\section{Importância do método}

"Pensar o Design sem a preocupação com a metodologia adequada a sua execução, na maioria das vezes, leva o projeto ao fracasso" (GOMEZ, PEREIRA E SCHLEMPER, 2010, p. 01). Tendo em vista a importância da metodologia para o design, buscaram-se as origens desta no discurso do método científico a fim de entender suas contribuições. 
A importância do método aplicado à pesquisa científica está em atenuar a influência da opinião própria do responsável pelo processo, a fim de maximizar a imparcialidade na experiência desenvolvida. Isso ocorre, devido ao conhecimento empírico, uma vez que o ser humano faz uso das informações com base nas suas experiências, crenças culturais e pessoais. A parcialidade é fator de empecilho, em qualquer situação, pois os resultados obtidos no meio científico servem como base para novas pesquisas e limitam assim, a assertividade.

A metodologia é o estudo dos métodos ligados à solução de problemas. "O conceito método deriva etimologicamente do greco-latino e significa caminho para alguma coisa, seguir ou andar ao longo de um caminho" (MERINO, GONTIJO E MERINO, 2011, p. 70)

Originariamente devida a Descartes, a metodologia propõe chegar à verdade através da dúvida sistemática e da decomposição do problema em pequenas partes, características estas que definiram a base da pesquisa científica (LANA, 2011, p. 55). No Discurso do Método, obra original datada de 1637, Descartes apresenta o método descrito através de quatro preceitos e que apontam claramente a eficácia do seu uso, para obter a verdade.

O primeiro era o de jamais acolher alguma coisa como verdadeira que eu não conhecesse evidentemente como tal; isto é, de evitar cuidadosamente a precipitação e a prevenção, e de nada incluir em meus juízos que não se apresentasse tão clara e distintamente a meu espírito, que eu não tivesse nenhuma ocasião de pô-lo em dúvida.

O segundo, o de dividir cada uma das dificuldades que eu examinasse em tantas parcelas quantas possíveis e quantas necessárias fossem para melhor resolvê-las.

O terceiro, o de conduzir por ordem meus pensamentos, começando pelos objetos mais simples e mais fáceis de conhecer, para subir, pouco a pouco, como por degraus, até o conhecimento dos mais compostos, e supondo mesmo uma ordem entre os que não se precedem naturalmente uns aos outros.

E o último, o de fazer em toda parte enumerações tão completas e revisões tão gerais, que eu tivesse a certeza de nada omitir. (DESCARTES, $1973,2^{\circ}$ parte) 
Segundo Moraes \& Mont'Alvão (2009), o pesquisador procura conhecer e interpretar a realidade, interessa-se em descobrir e observar fenômenos e procura descrevê-los, classificá-los e interpretá-los. Sendo assim, a função do método é proporcionar um padrão e um direcionamento objetivo no desenvolvimento de estudos, produzindo resultados eficazes.

As características descritas no método foram sendo rearranjados a fim de cumprir seu papel nas diversas áreas de conhecimentos. Para esse estudo deverá ficar claro a função do método aplicado ao design. A atividade profissional de design é metodológica e caracteriza-se por uma série de procedimentos reconhecidos pela academia científica, "sem o método, o que resulta não é design, mas o acaso, que não permite valoração quantitativa e ou qualitativa, repetição ou aprimoramento". (LANA, 2011, p. 60). Para Moraes (2011), as metodologias, cada vez mais, se sistematizam para levar em consideração fatores psicológicos, semânticos, da interface e até mesmo de sentimentos, visando desenvolver produtos que satisfaçam às necessidades do homem moderno com base em metodologias mais eficazes.

\section{Gestão de design}

Segundo моzота (2011), o processo de Gestão do Design teve sua origem datada na década de 60 na Grã-Bretanha. O termo inicialmente referia-se ao gerenciamento das atividades resultantes da relação agência de design x clientes.

Gestão do Design, segundo Wolf (1998), é a organização e coordenação de todas as atividades de design, baseada nos objetivos e valores definidos pela empresa. Deve fazer parte da missão da empresa e a premissa básica para sua eficiência é a consciência e a aceitação do design como fator de qualidade e de estratégia pela diretoria.

Para Martins e Merino (2011), a importância da gestão do design como processo e ferramenta estratégica, está na contribuição do design para o surgimento de um produto. A importância do mesmo é variável com as dimensões da organização, o ciclo de vida do produto e outras variáveis.

O design funciona como ponte entre a empresa/organização e o cliente. Buscar contribuições no produto, no tempo de concepção e produção, na concepção de uma identidade visual, no fortalecimento de uma imagem coorporativa e propor melhorias através da ergonomia, nas condições de trabalhos, são estratégias de aproximação com o público-alvo. O design visa evidenciar os valores de uma instituição, com o propósito final de atingir a sociedade - o design, em uma visão mais filosófica, entende de pessoas, busca propiciar condições favoráveis para a execução de um projeto, para uma experiência de uso 
a fim de, gerar satisfação por parte de todos os envolvidos em uma atividade, produto ou serviço.

A Gestão do Design tem como sua principal característica, a multidisciplinaridade e busca através disso, integrar o processo de desenvolvimento de um produto ou serviço, desde a criação ou concepção inicial até a comercialização do mesmo, visando atender as necessidades do consumidor e, além disso, satisfazer a organização e os seus colaboradores. Implementar a Gestão do Design consiste em uma mudança comportamental, através de trabalho co-participativo, estruturação de imagem corporativa e acima de tudo, significa estabelecer metas e diretrizes para que a organização tem um plano de conduta a longo prazo.

Para GORD (1990, apud mozota, 2011), a Gestão do Design "é a distribuição eficaz, pelos gerentes, dos recursos disponíveis à empresa a fim de ajudá-la a atingir seus objetivos". Assim, pensar em Gestão do Design, significa direcionar-se ao posicionamento do design em uma instituição ou organização, preocupando-se constantemente com os apontamentos da área relevantes a solução de questões gerenciais e no treinamento de gestores e demais integrantes da instituição em prol do desenvolvimento da mesma.

\section{Abordagem sistêmica como método}

Toma-se como base a visão do Design inserido no processo artesanal - os processos de detecção serão feitos levando em consideração os aspectos estéticos, o processo de fabricação e o tempo de processo, o desenvolvimento, a seleção de matéria-prima e a capacitação dos artesãos. O diagnóstico será produto da Abordagem Sistêmica entre o Design e o Artesanato, o arranjo levará em consideração os aspectos estratégicos, táticos e operacionais, enfatizando na visão estratégica direcionada ao processo operacional do artesanato.

De modo geral, os resultados do processo metodológico seguem o esquema abaixo, no qual o design se une ao artesanato (foco de estudo) e são pontuados dentro dos três níveis de ação: estratégico, tático e operacional.

A utilização da abordagem sistêmica como método, direciona o design de uma forma aplicada as necessidades do sistema e soluciona os problemas dentro de uma instituição ou grupo organizacional de uma forma macro, interrelacionando os atores do arranjo sistemático e seus fatores condicionantes na medida em que se aumenta a sua complexidade. Essa abordagem sistemática não tem relação somente com os aspectos físicos do sistema, mas também com a informação, que desempenha uma função essencial no processo de comunicação com os usuários (HESKETT, 2008, p. 106). 


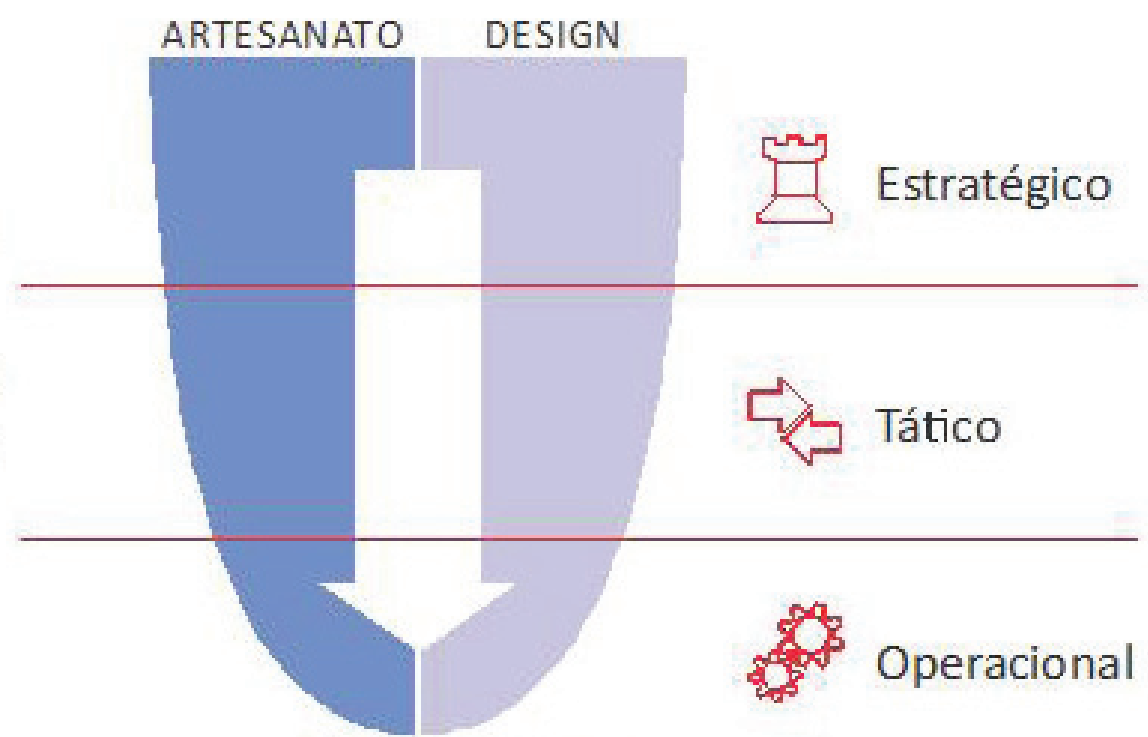

\section{GESTĀO DE DESIGN}

Figura 1. Níveis de ação

E, quando falamos em informação, entende-se por organização de dados, que por sua vez, configuram-se como observações provenientes do mundo:

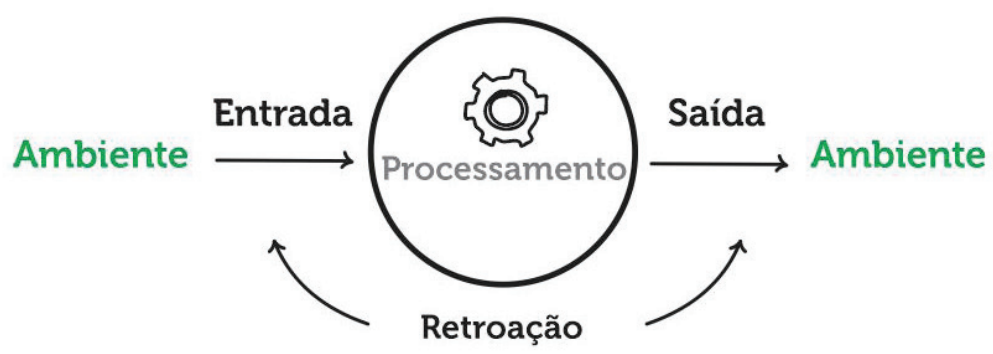

Figura 2. Sistematização da informação

\section{Artesanato}

O termo artesanato, surge do latim ARs, capacidade de fazer alguma coisa e que, mais tarde, passou a significar: arte. O profissional atuante ou que executa o processo, denomina-se artesão e é clara definição, é aquele que faz manualmente (por sua conta) objetos para uso doméstico.Esses objetos de uso doméstico configuram-se como um produto artesanal ou artefato artesanal que são resultado de uma atividade prática desenvolvida através da habilidade manual do artesão com nenhum ou auxílio parcial, de ferramentas. 
O artesanato é uma das manifestações culturais de um povo e sua revitalização tem efeitos que vão muito além da criação de oportunidades econômicas aos membros da comunidade envolvidos com a produção e comercialização de peças artesanais. O desenvolvimento do artesanato local tem efeitos na autoestima de uma sociedade e pode ser usado como complementação do produto turístico das destinações, já que há uma tendência dos turistas valorizarem peças originais e produzidas com técnicas locais. Configura-se como artesanato, toda atividade produtiva que resulte em objetos e artefatos acabados, feitos manualmente ou com a utilização de meios tradicionais ou rudimentares, com habilidade, destreza, qualidade e criatividade.

Segundo Albuquerque (2005), o termo "design social", representa a forte interação entre o design e o artesanato. Porém, esta interação deve ser bastante cuidadosa, já que não se pode impor aos artesãos regras meramente mercadológicas. É importante que o design complemente o artesanato de uma forma adequada, fazendo, por exemplo, com que a produção de artefatos seja mais competitiva e apresente melhor uso de materiais, cores, embalagens e etc.

A criatividade que caracteriza o povo brasileiro e os artesãos e artistas populares em particular, os materiais utilizados e as técnicas empregadas na confecção dos produtos artesanais, traduzem a sua identidade e a riqueza da sua cultura. Além de materializar a alma da cultura brasileira, o artesanato é um setor da economia cujo crescimento possui alto potencial de geração de trabalho e renda, merecendo uma política de desenvolvimento sustentável, levando à preservação das culturas locais e à formação de uma mentalidade empreendedora, por meio da capacitação das organizações e de seus artesãos para a sociedade de mercado, onde o padrão de qualidade e a capacidade de produção elevariam a aceitação e competitividade do produto.

Dessa forma, se faz necessário a adoção de uma cultura empresarial, caracterizando as ações de fomento a atividade artesanal e a valorização do artesão, através de iniciativas que integrem o setor público, privado e a sociedade civil e de políticas públicas para o seu desenvolvimento, inserindo o artesanato na economia real.

A partir das definições e estruturações do conceito de artesanato, desenvolveu-se uma mandala que se subdivide-se em aspectos de conceituação, funcionalidade, categorização, organização, matéria-prima utilizada e até mesmo no que não pode ser artesanato: 


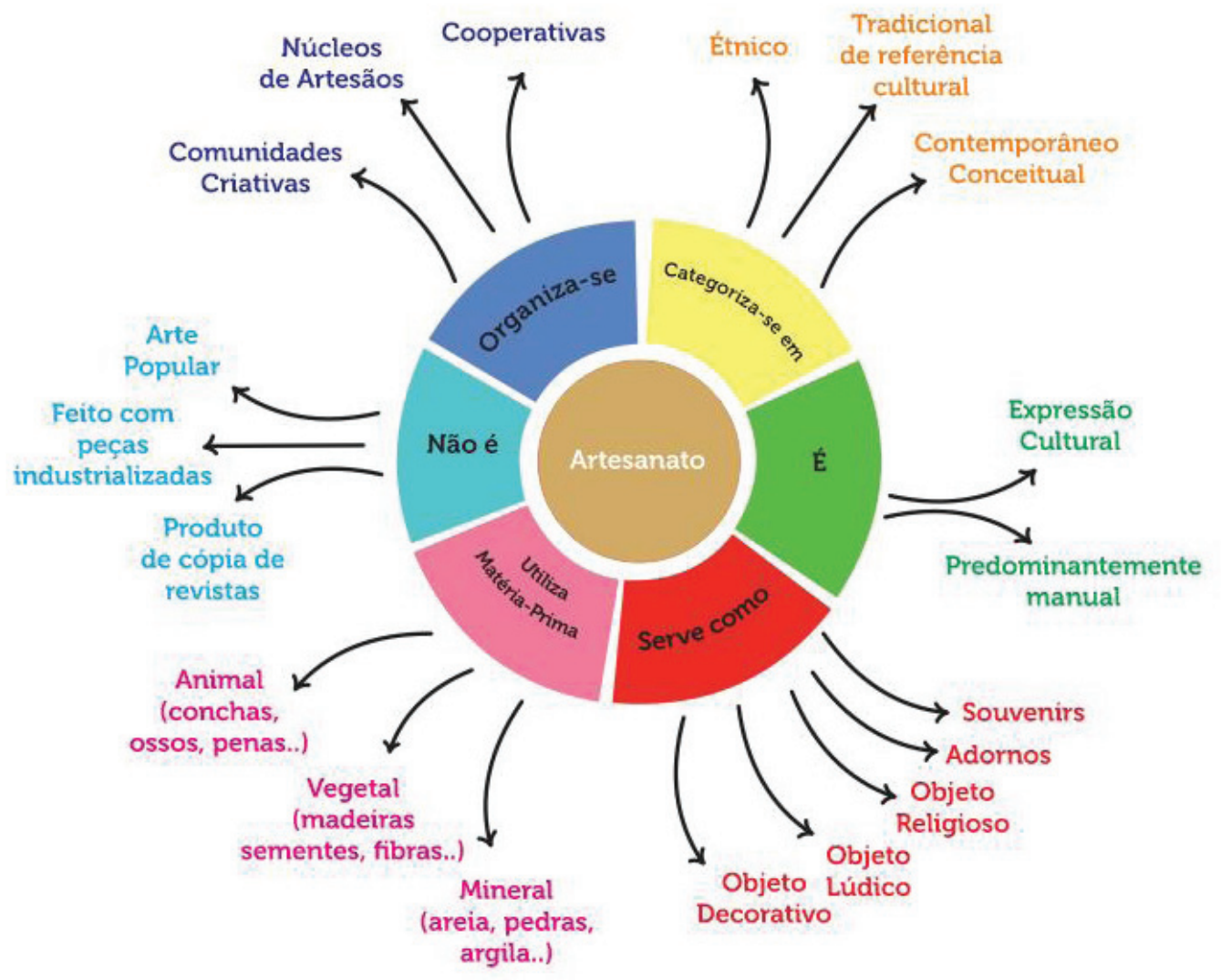

Figura 3. Mandala conceitual do artesanato

\section{Comunidades criativas}

Comunidade criativa consiste na organização em grupos de indivíduos que buscam desenvolver sistemas facilitadores como alternativas às soluções "institucionalizadas" para resolverem seus problemas cotidianos. Existe um número crescente de pessoas que se organizam para arranjarem soluções para alguns dos novos problemas com que a sociedade contemporânea é confrontada. A estas pessoas podemos chamar empreendedores sociais ou comunidades criativas. Empreendedores sociais são indivíduos que apresentam soluções inovadoras para os problemas sociais. São ambiciosos e persistentes, e contestam os grandes problemas sociais, oferecendo novas ideias para uma mudança global.

Em vez de deixarem este tipo de problemas encarregues ao setor governativo ou a negócios privados, detectam o que não funciona e resolvem o problema modificando o sistema, difundindo a solução e persuadindo outros grupos de pessoas a fazerem o mesmo. 
Segundo Manzini (2008), as comunidades criativas são grupos de pessoas inovadoras que se organizam para resolverem um problema, ou para abrirem novas possibilidades, e ao agirem desta forma, dão um passo positivo no processo de aprendizagem da sustentabilidade social e ambiental.

O que existe de comum entre os empreendedores sociais e as comunidades criativas é o fato de serem pessoas normais que inventam novas maneiras de ultrapassarem os problemas quotidianos e de participarem na vida pública de uma forma ativa.

Esta atividade, não muito comum numa sociedade caracterizada, sobretudo pela passividade, resulta de uma vontade de mudança sem esperar que sejam as instituições tradicionais a resolverem os problemas. E esta mudança é posta em prática por pessoas criativas que persistem e investem nas suas próprias ideias, e que são capazes de transformá-las em serviços inovadores, respondendo a necessidades do dia-a-dia. Estas ideias são designadas como inovações sociais, isto é, novas ideias que funcionam para responder a necessidades não satisfeitas e melhorar a vida das pessoas.

As oportunidades geradas por estas inovações parecem estar em consonância com o desenvolvimento sustentável, seja a nível ambiental, seja a nível social.

Considerando que o design teve um importante papel na criação do existente sistema de consumo, busca-se uma reinvenção desse mesmo sistema, mas baseado em modelos sustentáveis. Esta reinvenção poderia ser feita através do uso das inovações sociais e dos serviços criados a um nível das bases, ou readaptando as iniciativas top-down, que no seu formato original fracassaram ao darem resposta às exigências atuais.

Desenhar a vida das pessoas não se enquadra no âmbito da atividade do designer, nem é tão pouco uma opção desejável. A abordagem criativa à renovação e revitalização de redes sociais implica a participação ativa das pessoas no projeto de design. O papel do designer é de trabalhar lado a lado com as pessoas, com as suas necessidades e aspirações. Se as pessoas são capazes, de algum modo, de projetar a sua vida, a figura do designer será a de trabalhar com elas de forma a estruturar e potenciar as suas soluções criativas e, muitas vezes inovadoras. E este processo deveria resultar num outro muito mais fértil, uma vez que os "utilizadores" têm uma percepção mais clara das suas necessidades, e o designer, uma abordagem mais objetiva à resolução de problemas. 


\section{Diagrama de visualização geral}

O design é melhor meio para construir um relacionamento indireto com clientes, criando valor nas vidas dos mesmos, é a forma mais viável pra criar uma conexão entre o produto e o consumidor.

De um modo geral, o propósito é único: identificar visualmente os produtos inseridos tornando-os únicos e diferenciados. A identidade visual é tão relevante quanto sua função e a identidade é entendida como resultado final de decisões mercadológicas, funcionais e emocionais.

O diagrama de visualização busca apresentar uma visão macro do comportamento do sistema - apresentando os principais atores e suas áreas de interação. No sistema estudado, apresenta-se o nível enquanto sociedade cujo estão inseridos os atores: empreendedores e designers e o nível enquanto comunidade criativa cujos integrantes desenvolvem o artefato artesanal que resultará ou não, em uma expressão cultural. Os empreendedores enquanto atores, apenas apresentam suas intenções e necessidades e aguardam o retorno da comunidade criativa e futuramente o resultado do produto final; o designer, por sua vez, busca um limiar de intersecção para que possa acompanhar o processo e intervir, sem afetar na gênese cultural do desenvolvimento do processo do artefato. A comunidade criativa então, aplica a informação adquirida aliada ao conhecimento tácito e desenvolve o artefato:

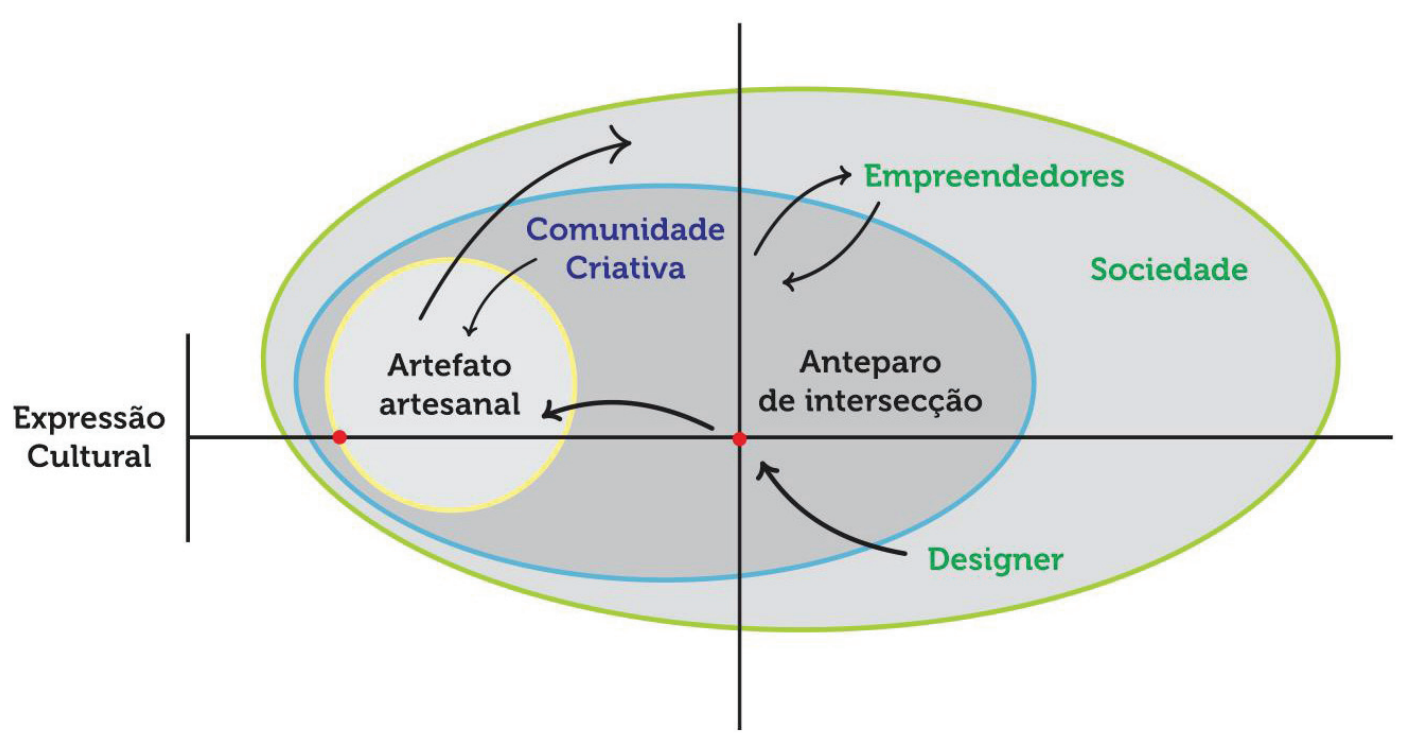

Figura 4. Diagrama de visualização geral 


\section{Considerações finais}

O artigo procura contribuir de forma significativa com o processo de produção artesanal desenvolvido em comunidades criativas, objetivando intensificar a aplicação de atividades sustentáveis na concepção de artefatos.

Ao mesmo tempo em que se fortalece a preocupação da geração de uma identidade consolidada para os produtos artesanais e torna esta imagem de qualidade visual, funcional e estética, ressalta-se também a integração entre a Gestão de Design e o Artesanato.

Além de contribuir positivamente para o desenvolvimento de comunidades criativas, a intenção é fornecer diretrizes passíveis de serem replicadas em outros modelos a fim de contribuir para o desenvolvimento organizacional do sistema estudado e suas respectivas atividades. Isso se dá, ao mesmo tempo, em que se busca firmar e mostrar os objetivos e as necessidades de uma forma clara, concisa e visualmente agradável através da utilização de esquemas, mapas conceituais, diagramas ou qualquer representação gráfica, que apresente um embasamento teórico definido.

\section{Referências}

CANCLINI, N. As culturas populares no capitalismo. São Paulo: Brasiliense, 1983.

DESCARTES, René. Discurso do método. São Paulo: Abril Cultura, 1973. Col. Os Pensadores, vol. XV.

GIL, Antônio Carlos. Métodos e técnicas de pesquisa social. 5. ed. - São Paulo: Atlas, 1999

GOMEZ, Luiz Salomão Ribas. PEREIRA, Alice Theresinha Cybis. SCHLEMPER, Paula Felipe. Os 4p's do Design: Uma proposta metodológica não linear de projeto. 2010. Disponível em: http://pt.scribd.com/doc/36684758/4-P-sdo-Design. Acesso: 15/06/12

HESKETT, John. Design / John Heskett; revisão técnica Pedro Fiori Fernandes; [tradutora Márcia Leme]. São Paulo: Ática, 2008. 
LANA, Sebastiana Luiza Bragança. A complexidade dos métodos em design. Cadernos de Estudos Avançados em Design: Método-Barbacena, MG: EdUEMG, 2011 - p. 53-65

MANZINI, E. "Design para a inovação social e sustentabilidade". Cadernos do Grupo de Altos Estudos, Programa de Engenharia de Produção da Coppe/ UFRJ, Rio de Janeiro, vol. I, 2008.

MARTINS, Rosane Fonseca de Freitas, MERINO, Eugenio Andrés Díaz. Gestão de Design como Estratégia Organizacional. 2º ed. Londrina: EDUEL, 2011.

MERINO, Giselle; Gontijo, Leila Amaral; Merino, Eugenio. O percurso do Design: no ensino e na prática. Cadernos de Estudos Avançados em Design: Método - Barbacena: EdUEMG, 2011. p.67-85.

MORAES, Anamaria de; MONT'ALVÃO, Claúdia. Ergonomia: Conceitos e Aplicações - Rio de Janeiro: 2AB, 2009.

MORAES, Dijon De; DIAS, Regina; BOM CONSELHO, Rosemary. Cadernos de Estudos Avançados em Design: Método - Barbacena, MG: EdUEMG, 2011.

MOZOTA, Brigitte Borja de; KLOPSCH, Cássia; COSTA, Filipe Campelo Xavier da. Gestão de Design: usando o design para construir valor de marca e inovação corporativa. Porto Alegre: Bookman, 2011. 
Recebido em: 10/06/2013

Aceito em: 01/10/2013

\section{Como citar}

SANTOS, Érik Silva dos; FIGUEIREDO, Luiz Fernando Gonçalves de; MERINO, Giselle Schmidt Alves Diaz. A interação em estudo: as análises das correntes teóricas sobre o conceito de design de interação para profissionais e educadores. Arcos Design. Rio de Janeiro: PPD ESDI - UERJ. Volume 7 Número 2 Dezembro 2013. pp. 53-68. Disponível em: [http://www.e-publicacoes.uerj.br/ index.php/arcosdesign]

\section{DOI}

10.12957/arcosdesign.2013.12181

\section{(c) (†)}

A Revista Arcos Design está licenciada sob uma licença Creative Commons Atribuição - Não Comercial - Compartilha Igual 3.0 Não Adaptada. 